\title{
p.Arg75GIn, a CFTR variant involved in the risk of CFTR-related disorders?
}

\author{
Brigitte Martinez ${ }^{1}$, Marion Heller ${ }^{1}$, Natacha Gaitch ${ }^{1}$, Dominique Hubert ${ }^{2}$, Pierre-Regis Burgel ${ }^{2}$, \\ Philippe Levy ${ }^{3}$, Emmanuelle Girodon ${ }^{1}$ and Thierry Bienvenu ${ }^{1,4}$
}

c.224G $>$ A, p.Arg75GIn (R75Q) presumably leads to an amino-acid change from arginine to glutamine in the membranespanning domain of the CFTR protein. Initially reported as a benign sequence variation, p.Arg75GIn was shown to be associated with a high risk of pancreatitis, a risk that was strikingly higher when p.Arg75GIn was combined with a SPINK1 variant. In addition, it was shown that p.Arg75GIn alters bicarbonate but not chloride conductance and that the mutation also induces exon 3 skipping. To investigate the role of p.Arg75GIn in idiopathic chronic pancreatitis (ICP), we performed genotyping of the CFTR gene in 880 patients with ICP, 198 patients with idiopathic bronchiectasis (IB), 74 patients with classical cystic fibrosis (CF), 48 patients with congenital bilateral absence of the vas deferens (CBAVD) and 148 healthy controls. p.Arg75Gln variant was identified in $3.3 \%(29 / 880)$ of patients with ICP, 3.3\% (9/272) patients with a pulmonary disease, $2.1 \%(1 / 48)$ of patients with CBAVD and $4.7 \%(7 / 148)$ of healthy controls. It was frequently associated with the c.[1210-12T[7];1408A $>$ G] (T7-p.Val470) allele and this CFTR genetic background could not explain the putative pathogenicity of this variant. To assess whether CFTR and SPINK1 mutations are co-inherited in pancreatitis, we sequenced SPINK1 gene exon 3 in the 46 patients who were previously identified to be heterozygous for p.Arg75GIn. Two SPINK1 pancreatitis-associated variants, p.Asn34Ser and p.Pro55Ser, were found in 6 patients: 4 of 29 (13.8\%) patients with ICP (3 p.Asn34Ser and 1 p.Pro55Ser), 1 of 7 (14.3\%) healthy controls (p.Asn34Ser) and 1 of $9(11.1 \%)$ patients with IB (p.Pro55Ser). Our study does not confirm that the CFTR p.Arg75GIn mutation confers a significant risk of pancreatitis both when considered individually and with a concurrent SPINK1 mutation, suggesting the role of other genetic and environmental factors.

Journal of Human Genetics (2014) 59, 206-210; doi:10.1038/jhg.2014.2; published online 23 January 2014

Keywords: CFTR; cystic fibrosis; p.Arg75GIn; pancreatitis; SPINK1

\section{INTRODUCTION}

The p.Arg75Gln (R75Q) mutation is a consequence of a nucleotide change from guanine to adenine at position c.224 in the Cystic Fibrosis Transmembrane conductance Regulator (CFTR) gene (NM_000492.3), which presumably leads to an amino-acid change from arginine (Arg) to glutamine (Gln). A basic polar amino-acid change is substituted with a neutral polar amino acid in a membranespanning domain of the CFTR protein. Initially, Zielenski et al. ${ }^{1}$ reported p.Arg75Gln as a neutral benign sequence variation that was not involved in cystic fibrosis (CF). In 2008, Gené et al. ${ }^{2}$ evaluated the impact of this p.Arg75Gln missense change on the channel behaviour and found that p.Arg75Gln had a glycosylation pattern and a subcellular distribution comparable to the wild-type (WT) CFTR, and that the channel activity of p.Arg75Gln analysed by singlechannel patch-clamp analyses appeared similar to that of the WT CFTR. These biochemical and biological arguments suggested that p.Arg75Gln is a sequence variant that does not contribute to the autosomal recessive disorder CF. However, the fact that p.Arg75Gln was frequently reported in a number of individuals with CFTR-related disorders (CFTR-RD) such as bronchiectasis, congenital bilateral absence of vas deferens, asthma, chronic obstructive pulmonary disease and pancreatitis suggested that normal function may be disrupted in some way. ${ }^{3-7}$ Recently, to assess the physiological properties of the p.Arg75Gln variant, Schneider et al. ${ }^{8}$ stably expressed mutant and WT CFTR in HEK293 cells and tested the relative conductance of each CFTR protein to bicarbonates and chloride. The current ratio at $60 \mathrm{mV}$ in $\mathrm{Cl}^{-}$media was 0.76 for p.Arg75Gln, which was not statistically different from 1.0 for the wild type (WT). However, the current ratio in bicarbonate media for p.Arg75Gln was 0.18 and the $\mathrm{HCO} 3^{-} / \mathrm{Cl}^{-}$current ratio for p.Arg75Gln was 0.053, four times lower than that for CFTR WT. These data showed for the first time that p.Arg75Gln alters bicarbonate but not chloride conductance. More recently, it has been shown that the c.224G $>$ A substitution (p.Arg75Gln) induced a

${ }^{1}$ Laboratoire de Biochimie et Génétique Moléculaire, GH Cochin-Broca-Hôtel Dieu, AP-HP, Paris, France; ${ }^{2}$ Service de Pneumologie, GH Cochin-Broca-Hôtel Dieu and Université Paris Descartes, Sorbonne Paris Cité, Paris, France; ${ }^{3}$ Pôle des Maladies de l'Appareil Digestif, Service de Gastroentérologie-Pancréatologie, Hôpital Beaujon, AP-HP, Université Paris VII-Denis Diderot, Clichy, France and ${ }^{4}$ Institut Cochin, INSERM U1016, Université Paris Descartes Paris, Paris, France

Correspondence: Professor T Bienvenu, Génétique et Développement, Institut Cochin, INSERM U1016, 24 rue du Faubourg Saint Jacques, Paris 75014, France. E-mail: thierry.bienvenu@inserm.fr

Received 17 October 2013; revised 2 December 2013; accepted 5 December 2013; published online 23 January 2014 
four- to fivefold increase in exon 3 skipping of the CFTR gene, suggesting that it is a splicing mutation. ${ }^{9}$ Interestingly, Schneider et al. $^{8}$ showed a very high risk of pancreatitis in American patients with p.Arg75Gln (odd ratio, 3.4) and a striking increase in risk of pancreatitis when CFTR p.Arg75Gln was combined with a SPINK1 variant (odd ratio, 25.1). However, in 2013, Rosendahl et al. ${ }^{10}$ examined CFTR as well as other known pancreatitis-causing genes in a large cohort of German patients with chronic pancreatitis (CP) (107 hereditary pancreatitis (HP), 553 ICP) and in controls (1758 healthy individuals), and found similar frequencies of the p.Arg75Gln variant in patients with HP or ICP and in controls.

To unravel discrepancies between these previous reports, and in order to determine whether or not the p.Arg75Gln variant affects the risk of developing a CFTR-RD, we performed the complete screening of the CFTR gene in 1348 individuals distributed in four different groups of patients (ICP, CF, IB and congenital bilateral absence of the vas deferens (CBAVD)) and ethnically matched controls. We also looked for SPINK1 gene mutations in patients or subjects bearing the p.Arg75Gln variant under the hypothesis of an impact of the double heterozygous carriage of CFTRp.Arg75Gln and SPINK1 p.Asn34Ser.

\section{MATERIALS AND METHODS}

\section{Patients}

The study protocol was reviewed and approved by the institutional review board for clinical research of Assistance Publique-Hopitaux de Paris and the ethics committee of the Medical Faculty of Paris Descartes University. Written informed consent was obtained from all participants. Five groups of individuals were studied: 880 unrelated patients with ICP, 74 patients with a true diagnosis of CF, 198 patients IB, 48 patients with CBAVD and 148 healthy French subjects of Caucasian origin who requested genetic counselling because their partner was a patient with CF (with two identified CF-causing mutations).

The diagnosis of $\mathrm{CP}$ was based on the presence of at least one of the following: pancreatic calcifications as evidenced by CT scan or endoscopic ultrasonography; moderate to marked pancreatic ductal lesions on pancreatography obtained by MRCP (Cambridge classification) or typical histology of an adequate surgical pancreatic specimen. Evident causes of acute recurrent pancreatitis or CP were ruled out: biliary lithiasis (presence of gallbladder, common bile duct lithiasis or sludge at any imaging procedure including endosonography, hypercalcemia $\left(>3 \mathrm{mmoll}^{-1}\right)$, hypertriglyceridemia $\left(>10 \mathrm{mmoll}^{-1}\right)$, drugs/medication causing acute pancreatitis, personal history of abdominal radiotherapy, autoimmune disease (typical imaging, personal history of autoimmune disease, dosing of gammaglobulin, immunoglobulin A, G (including subfraction IgG4) and M, Latex Waaler-Rose, anti-nuclear antibodies, anti-smooth muscle antibodies, anti-mitochondrial antibodies, anti-neutrophil cytoplasmic antibodies), traumatic pancreatitis and duct obstruction. Excess alcohol intake was considered as a cause of pancreatitis if pure alcohol consumption exceeded $60 \mathrm{~g}$ per day for $>2$ years).

Patients with a pulmonary disease (CF and IB) were assessed according to standards proposed by European Respiratory Society. A diagnosis of bronchiectasis was established by high-resolution computed tomography of the chest and a specific aetiology of the bronchiectasis was undetermined at study entry. Subjects with known immune compromised states, current therapy with immune modulating therapies and known malignancy were excluded. Laboratory evidence of $\mathrm{CF}$ was based on sweat chloride values and/or a true CF genotype.

The clinical diagnosis of CBAVD was based on clinical examination with impalpable vas deferens, transrectal, ultrasonography, semen analysis (volume, $\mathrm{pH}$ and sperm count in accordance with the World Health Organization guidelines) and low concentration of fructose and citrate. Patients with renal abnormalities were excluded.

Healthy individuals were recruited in the region of the study and were well matched for potential confounders such as environmental factors and ethnicity.

\section{Methods}

For CFTR gene analysis, a $10 \mathrm{ml}$ blood sample was drawn from each of the participants. Genomic DNA was extracted from nucleated cells by a standard technique (Wizard Genomic DNA purification kit, Promega, Madison, WI, USA). We tested for the 32 most frequently observed CFTR mutations with the CF assay kit (v3 Genotyping assay, Abbott, Wiesbaden, Germany). This kit detects $\sim 83 \%$ of the mutations observed in French CF patients. A complementary analysis of the whole coding region and exon/intron junctions of CFTR was carried out systematically, by denaturing gradient gel electrophoresis (DGGE) (exons 9 and 10) and denaturing high-performance liquid chromatography (DHPLC) (all other exons). This approach detects $>95 \%$ of the CFTR mutations and variants in our CF population. Mutations and variants detected by DGGE and DHPLC were subsequently confirmed by direct DNA sequencing with the ABI PRISM Big Dye Terminator cycle sequencing kit (Applied Biosystems, Foster City, CA, USA) and an ABI Prism 3100-Avant sequencer. If the p.Arg75Gln variant was detected, we screened exon 3 of the SPINK1 gene by direct sequencing on an ABI3130 sequencer using the Big Dye Terminator Cycle sequencing kit as previously described. ${ }^{8,10}$ Mutation numbering was based on cDNA numbering (nucleotide +1 corresponds to the A of the ATG translation initiation codon of the relevant GenBank mRNA reference NM_003122.1 (CFTR gene, NM_000492.3) and of the SPINK1 genomic reference sequence (NT_029289.10). Traditional mutations names are also indicated in brackets at their first occurrence. Exon numbering followed Human Genome Variation Society (HGVS) recommendations (exons 1-27).

\section{RESULTS}

Numerous CF-causing and CFTR-RD-associated mutations were found in the groups of patients. Notably, among 880 ICP patients, we identified a total number of 264 mutant CFTR alleles (15\% of chromosomes), in 220 patients (Table 1). Forty-four patients (5.0\%) were compound heterozygous for two mutations, either one $\mathrm{CF}$ or one CFTR-RD or two CFTR-RD mutations. In the group of 148 control subjects, three were heterozygous for a CF-causing mutation (1 c.2125C > T (R709X), 2 c.1521_1523delCTT (F508del)); 7 carried c.224G $>$ A (R75Q); 12 carried CFTR-RD associated mutations

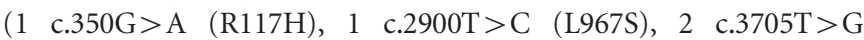
(S1235R), 1 c.2991G $>$ C $\quad($ L997F $), \quad 1 \quad$ c. $[220 \mathrm{C}>\mathrm{T} ; 3808 \mathrm{G}>\mathrm{A}]$ (R74W;D1270N), 2 c.[1727G >C;2002C > T] (G576A;R668C), 1 c. $1523 \mathrm{~T}>\mathrm{G} \quad$ (F508C), 1 c.1865G $>$ A $\quad$ (G622D), 1 c.332C $>$ T (P111L), 1 c.2855T $>$ C (M952T)); 2 carried potential splicing mutations $\quad\left(\begin{array}{ll}1 & \text { c. } 3717+45 \mathrm{G}>\mathrm{A}\end{array} \quad(3849+45 \mathrm{G}>\mathrm{A}), \quad 1 \quad\right.$ c.3139+ $18 \mathrm{C}>\mathrm{T} \quad(3271+18 \mathrm{C}>\mathrm{T})) ; \quad 7$ carried c. $1584 \mathrm{G}>\mathrm{A} \quad(1716 \mathrm{G}>\mathrm{A})$; finally, the c.1210-12T[5] variant was found in additional 11 subjects (4 c.1210-34TG[12]T[5] and 7 c.1210-34TG[11]T[5]). In the ICP

Table 1 CFTR genotypes and alleles in ICP patients and controls

\begin{tabular}{lccc}
\hline & $\begin{array}{c}\text { ICP Patients } \\
(\mathrm{n}=880)\end{array}$ & $\begin{array}{c}\text { Controls } \\
(\mathrm{n}=148)\end{array}$ & \\
CFTR genotypes and alleles & $\mathrm{n}(\%)$ & $\mathrm{n}(\%)$ & P-value* \\
\hline Compound heterozygous genotypes & $26(2.95)$ & $0(0.0)$ & 0.06 \\
excluding c.1210-12T[5] & & & \\
Compound heterozygous genotypes & $18(2.04)$ & $1(0.67)$ & 0.41 \\
including c.1210-12T[5] & & & \\
CF-causing mutations & $52(5.9)$ & $3(2.0)$ & 0.08 \\
CFTR-RD mutations & $83(9.43)$ & $12(8.10)$ & 0.71 \\
c.1210-34TG[11]T[5] & $64(7.27)$ & $7(4.72)$ & 0.34 \\
c.1210-34TG[12]T[5] & $33(3.75)$ & $4(2.70)$ & 0.68 \\
c.1210-34TG[13]T[5] & $3(0.34)$ & $0(0.0)$ & 0.92 \\
C.224G $>$ A, p.Arg75GIn & $29(3.29)$ & $7(4.72)$ & 0.25 \\
c.1584G $>$ A & $50(5.68)$ & $7(4.72)$ & 0.77 \\
\hline
\end{tabular}

* $P$-values $<0.05$ are considered statistically significant. 
Table 2 CF or CFTR-RD associated mutations found in patients also carrying c.224G $>$ A, p.Arg75GIn (R75Q)

\begin{tabular}{lcccc}
\hline CF orCFTR-RD mutations & $\begin{array}{c}\text { ICP Patients } \\
(\mathrm{n}=29)\end{array}$ & $\begin{array}{c}\text { IB } \\
(\mathrm{n}=9)\end{array}$ & $\begin{array}{c}\text { CBAVD } \\
(\mathrm{n}=1)\end{array}$ & $\begin{array}{c}\text { Controls } \\
(\mathrm{n}=7)\end{array}$ \\
\hline $\begin{array}{l}\text { C.1521_1523del } \\
\text { (F508del) }\end{array}$ & 0 & 2 & 1 & 0 \\
$\begin{array}{l}\text { c.3194T>C (L1065P) } \\
\text { c.3140-26A }>\text { G }\end{array}$ & 0 & $1^{\mathrm{a}}$ & 0 & 0 \\
$\begin{array}{l}\text { (3272-26A>G) } \\
\text { c.1002-1110_1113del }\end{array}$ & 1 & 0 & 0 & 0 \\
c.2002C>T (R668C) & 1 & 0 & 0 & 0 \\
c.1210-34TG[12]T[5] & 0 & $1^{\text {a }}$ & 0 & 0 \\
\end{tabular}

Abbreviations: CBAVD, congenital bilateral absence of the vas deferens; IB, idiopathic bronchiectasis; ICP, idiopathic chronic pancreatitis.

Mutations names are indicated according to HGVS recommendations at the nucleotide level.

aThe patients carrying c.3194T >C (L1065P) and c.2002C > T (R668C) in addition to

p.Arg75GIn also carried c.1521_1523del (F508del).

group on the other hand, 26 patients carried two mutations and were potentially compound heterozygous. Although no control individual was compound heterozygous, the difference misses statistical significance (Table 1).

In our cohort of 1348 subjects, 46 individuals were heterozygous for the p.Arg75Gln variant (3.4\%). No p.Arg75Gln homozygote was identified. Among these individuals, seven were controls from the healthy population $(7 / 148,4.7 \%), 29$ were patients with ICP $(29 / 880$, $3.3 \%$ ), nine were patients with a pulmonary disease (74 CF and 198 IB) $(9 / 272,3.3 \%)$ and one was a patient with bilateral absence of the vas deferens $(1 / 48,2.1 \%)$. Among these 46 individuals bearing the p.Arg75Gln variant, only 8 carried another CFTR mutation (2/29 ICP patients, 5/9 IB patients and 1/1 CBAVD patient) (Table 2).

The frequency of the p.Arg75Gln in the ICP patients $(3.3 \%)$ and in the patients with CF or IB (3.3\%) was similar to that in the control group $(4.7 \%)$ (Table 3 ). The lower frequency of the p.Arg75Gln in CF $(0 / 74)$ and CBAVD patients $(2.1 \%)$ than in the control group $(4.7 \%)$ was mainly due to the presence of CF and CFTR-RD mutations that are not associated in cis with this amino-acid change.

As the CFTR genetic background could influence the potential pathogenicity of the p.Arg75Gln variant, we studied the association of the p.Arg75Gln mutation with the c.1408A $>$ G, p.Val470 allele in exon 11 and the c.1210-12T(5_9) (Tn repeat) located in intron 9. We found that the p.Arg75Gln was associated with the p.Val470 allele in 20 cases ( 15 ICP, 5 controls) and with the p.Met470 allele in only one case ( 1 control) (Table 4). In 25 cases, chromosome assignment was not possible (p.Met470 or p.Val470). We found that the p.Arg75Gln was associated with the c.1210-12T[7] allele in 32 cases (25 ICP, 1 IB, 6 controls) (Table 4). In 14 cases, chromosome assignment was not possible. p.Arg75Gln was associated with the c.[1210-34TG[11]T[7];1408A > G] (T7-p.Val470) in 19 cases (15 ICP, 4 controls) and with the c.[1210-34TG[11]T[7];1408A] (T7-p.Met470) in only one case (1 control) (Table 4). Our findings showed no evidence of a difference in the distribution of the c.1210-12T(5_9) alleles and in the frequency of p.Met470Val variant between the different groups of subjects who carried the p.Arg75Gln variant.

Among the 46 individuals bearing the p.Arg75Gln variant, four (8.7\%) were heterozygous for the p.Asn34Ser variant in the SPINK1 gene. One of them was from the healthy population $(1 / 7,14.3 \%)$ and three were from the ICP group $(3 / 29,10.3 \%)$. None of the patients with a pulmonary disease or with CBAVD bearing the p.Arg75Gln
Table 3 c.224G >A, p.Arg75GIn (R75Q) variant in CFTR-related disorders

\begin{tabular}{|c|c|c|c|c|c|c|}
\hline \multirow[b]{2}{*}{ Disease } & \multirow[b]{2}{*}{ Origin } & \multicolumn{2}{|c|}{ Patients with disease } & \multicolumn{2}{|c|}{ Control individuals } & \multirow[b]{2}{*}{ Study } \\
\hline & & $\begin{array}{l}\text { No. of } \\
\text { patients }\end{array}$ & $\begin{array}{c}\text { p.Arg75G/n } \\
\text { frequency }\end{array}$ & $\begin{array}{c}\text { No. of } \\
\text { individuals }\end{array}$ & $\begin{array}{c}\text { p.Arg75GIn } \\
\text { frequency }\end{array}$ & \\
\hline ICP & American & 56 & 1.8 & 50 & 4 & 13 \\
\hline ICP & American & 52 & 15.4 & 96 & 13.5 & 14 \\
\hline ICP & American & 80 & 16.25 & 95 & 9.5 & 8 \\
\hline ICP & German & 67 & 1.49 & 60 & 0 & 15 \\
\hline ICP & German & 20 & 10.0 & ND & 4.0 & 16,17 \\
\hline ICP & French & 253 & 4.7 & ND & ND & 18 \\
\hline HP & German & 107 & 4.7 & 1758 & 3.4 & 10 \\
\hline ICP & German & 553 & 3.3 & 1758 & 3.4 & 10 \\
\hline ICP & French & 880 & 3.3 & 148 & 4.7 & $\begin{array}{l}\text { This } \\
\text { study }\end{array}$ \\
\hline DB & Greek & 51 & 1.96 & ND & 1.9 & 19 \\
\hline DB & Italian & 120 & 0.83 & 68 & 0 & 20 \\
\hline DB & Serbian & 19 & 10.5 & ND & ND & 21 \\
\hline DB & American & 40 & 15.0 & ND & ND & 22 \\
\hline COPD & Serbian & 31 & 9.67 & ND & ND & 23 \\
\hline DB & French & 32 & 12.5 & ND & ND & 24 \\
\hline $\mathrm{DB} / \mathrm{IB}$ & French & 272 & 3.3 & 148 & 4.7 & $\begin{array}{l}\text { This } \\
\text { study }\end{array}$ \\
\hline Sarcoidosis & Italian & 52 & 7.76 & ND & ND & 25 \\
\hline Total & & 2685 & $\begin{array}{c}4.7 \\
(n=126)\end{array}$ & 2423 & $\begin{array}{c}3.75 \\
(n=91)\end{array}$ & \\
\hline
\end{tabular}

Abbreviations: CBAVD, congenital bilateral absence of the vas deferens; COPD, chronic obstructive pulmonary disease; $\mathrm{DB}$, diffuse bronchiectasis; $\mathrm{HP}$, hereditary pancreatitis IB, idiopathic bronchiectasis; ICP, idiopathic chronic pancreatitis.

Results are expressed as carrier frequency (number of individuals with at least one abnormal allele divided by the total number of individuals tested).

variant in the CFTR gene also carried the p.Asn34Ser variant in the SPINK1 gene.

Two patients (one with IB and one with ICP) carrying the p.Arg75Gln variant were also heterozygous for the p.Pro55Ser variant in the SPINK1 gene $(2 / 46,4.3 \%)$.

\section{DISCUSSION}

\section{Frequency of the p.Arg75Gln variant}

Initially, the p.Arg75Gln variant was considered as a benign variant. Its frequency in the general population was estimated to $1 \%$ in Northern Ireland and $2 \%$ in Serbia. ${ }^{11,12}$

Our findings confirm and extend the recent German report showing similar frequencies of the p.Arg75Gln in patients with pancreatitis $(3.3 \%$ and $4.7 \%$, respectively) and in controls $(4.7 \%$ and $3.4 \%$, respectively). ${ }^{10}$ p.Arg75Gln was also found at similar frequencies in patients with IB $(3.3 \%)$ and with CBAVD $(2.1 \%)$. We found no p.Arg75Gln in CF patients, mostly because all CF patients in this study were homozygous or compound heterozygous for CF-causing mutations that are not known to be associated with this variant.

This is entirely consistent with the observations of several other reports from patients with CFTR-RD such as ICP and diffuse bronchiectasis (Table 2) ${ }^{13-25}$ As our data contrast with other few previous reports, these observations raise the question of the pathogenicity of this amino-acid change in association with other genetic changes in CFTR-RD. ${ }^{8}$ 
Table 4 c.1210-34TG(9_13)T(5_9) and c.1408A $>$ G alleles found in association with c.224G $>A$, p.Arg75Gln (R75Q)

\begin{tabular}{|c|c|c|c|c|}
\hline p.Val/Met470; TGmTn repeat haplotype & $\begin{array}{c}\text { ICP } \\
\text { Patients } \\
(\mathrm{n}=29)\end{array}$ & $\begin{array}{c}I B \\
(\mathrm{n}=9)\end{array}$ & $\begin{array}{l}C B A V D \\
(\mathrm{n}=1)\end{array}$ & $\begin{array}{c}\text { Controls } \\
(\mathrm{n}=7)\end{array}$ \\
\hline $\begin{array}{l}\text { c.[1210-34TG[11]T[7];1408A > G] } \\
\text { (TG11T7-p.Val470) }\end{array}$ & 15 & & & 4 \\
\hline $\begin{array}{l}\text { c.[1210-34TG[11]T[7];1408A] } \\
\text { (TG11T7-p.Met470) }\end{array}$ & 0 & & & 1 \\
\hline $\begin{array}{l}\text { c.1210-34TG[11]T[7], c.1408A>G } \\
\text { undetermined (p.Val470 or p.Met470) }\end{array}$ & 1 & 1 & & 0 \\
\hline $\begin{array}{l}\text { c.1210-34TG[10]T[7] or TG[11]T[7], } \\
\text { c.1408A> G undetermined }\end{array}$ & 7 & & & 0 \\
\hline $\begin{array}{l}\text { c.1210-34TG[11]T[7] or TG[12]T[7], } \\
\text { c.1408A>G (p.Val470) }\end{array}$ & 0 & & & 1 \\
\hline $\begin{array}{l}\text { c.1210-34TG[11]T[7] or TG[12]T[7], } \\
\text { c.1408A > G undetermined }\end{array}$ & 2 & & & 0 \\
\hline Undefined haplotypes & 4 & 8 & 1 & 1 \\
\hline
\end{tabular}

Abbreviations: CBAVD, congenital bilateral absence of the vas deferens; IB, idiopathic bronchiectasis; ICP, idiopathic chronic pancreatitis.

\section{Effect of the CFTR genetic background on the pathogenicity of} p.Arg75Gln variant

We found that the p.Arg75Gln was frequently associated with the p.Val470 allele and the T7 allele. Our findings showed no evidence of a difference in the frequency of the c.1210-12T[7] allele and p.Met470Val between ICP and control groups of subjects who carried p.Arg75Gln. In the study of Divac et al. ${ }^{23}$, all patients with a chronic obstructive pulmonary disease bearing the p.Arg75Gln were also homozygous for p.Val470. It was shown that the p.Val470 CFTR protein has 1.7-fold less chloride activity and matures two times faster in comparison to the p.Met470 CFTR protein. Girodon et al. ${ }^{24}$ also found that the p.Arg75Gln variant associated with the TG11T7-p.Val470 allele in two cases (2/4). In other two cases, chromosome assignment was not possible. In three German cases with recurrent or chronic idiopathic pancreatitis, p.Arg75Gln was associated with the c.1210-12T[7]. ${ }^{15,16}$ p.Arg75Gln was observed in different genetic backgrounds in six American patients with bronchiectasis (1 c. [224G $>$ A;1210-12T[9], 1 c.[224G $>A ; 1210-12 T[7], 3$ c. [224G $>\mathrm{A} ; 1210-12 \mathrm{~T}[7] ; 1408 \mathrm{~A}>\mathrm{G}]$ and 1c.[224G $>\mathrm{A} ; 1210-12 \mathrm{~T}[9]$; $1408 \mathrm{~A}>\mathrm{G}]) .^{21}$ In total, our study confirms that p.Arg75Gln was frequently associated with the c.[1210-12T[7];1408A $>$ G] allele and shows that this CFTR genetic background cannot explain the putative pathogenicity of this variant.

\section{Effect of the p.Asn34Ser SPINK1 mutation on the pathogenicity of the p.Arg75Gln CFTR variant}

It is also plausible that the combination of the p.Arg75Gln CFTR variant with mutation(s) in one or more modifier genes could affect disease pathogenesis in some patients. For example, mutations in the SPINK1 gene, which are well-known risk factors for ICP, have been identified in some patients with pancreatitis who carry CFTR gene mutations on one allele. ${ }^{26}$ In a recent report, Schneider et al. ${ }^{8}$ observed a striking increase in the risk of pancreatitis by comparing the expected and the observed frequencies of the combination of the SPINK1 p.Asn34Ser variant with the CFTR p.Arg75Gln variant. Of 525 controls, only one subject $(0.19 \%)$ carried both variants and suffered from recurrent abdominal pain requiring hospitalisation and abdominal surgery. ${ }^{8}$ In contrast, 6 patients with ICP carried both variants $(6 / 80,7.5 \%)$, suggesting that coinheritance of CFTR p.Arg75Gln and SPINK1 p.Asn34ser significantly increases the risk of ICP.

However, in 2013, Rosendahl et al. ${ }^{10}$ examined CFTR as well as other known pancreatitis-causing genes (PRSS1, SPINK1 and CTRC) in a large cohort of German patients with CP (107 HP, 553 ICP) and in controls (1758 healthy individuals) and found similar frequencies of the p.Arg75Gln variant in patients with HP $(4.7 \%, 5 / 107)$ or ICP $(4.7 \%, 26 / 553)$ and in controls $(3.4 \%, 60 / 1758)$. Nevertheless, PRSS1, SPINK1 and CTRC variants (heterozygous and homozygous) were found in combination with CFTR p.Arg75Gln variant in 3/107 (2.8\%) patients with HP, in $8 / 553(1.44 \%)$ patients with ICP and in only $1 /$ $1758(0.06 \%)$ controls. The authors explained this unbalanced distribution of double heterozygotes by the significant overrepresentation of p.Asn34Ser in patients with pancreatitis $(16 \%$ $107 / 660$ vs $1.5 \% 26 / 1758$ controls). ${ }^{10}$

In conclusion, contrary to the findings of the report of Schneider et $a .^{8}$, we did not find any preferential coinheritance of CFTR p.Arg75Gln with SPINK1 p.Asn34Ser. Only three of the 29 CFTR p.Arg75Gln heterozygous patients with ICP were also SPINK1 p.Asn34Ser heterozygous. It is probable that other genes such as SLC26A9, SLC9A3 and SLC6A14 interplay with CFTR variants. ${ }^{27}$ Further molecular experiments need to be performed to confirm this hypothesis.

\section{CONFLICT OF INTEREST}

The authors declare no conflict of interest.

\section{ACKNOWLEDGEMENTS}

We thank the Association Vaincre La Mucoviscidose for financial support to the laboratory work.

1 Zielenski, J., Bozon, D., Kerem, B., Markiewicz, D., Durie, P., Rommens, J. M. et al. Identification of mutations in exons 1 through 8 of the cystic fibrosis transmembrane conductance regulator (CFTR) gene. Genomics 10, 229-235 (1991).

2 Gené, G. G., Llobet, A., Larriba, S., de Semir, D., Martínez, I., Escalada, A. et al. $\mathrm{N}$-terminal CFTR missense variants severely affect the behaviour of the CFTR chloride channel. Hum. Mutat. 29, 738-749 (2008).

3 Bombieri, C., Luisetti, M., Belpinati, F., Zuliani, E., Beretta, A., Baccheschi, J. et al. Increased frequency of CFTR gene mutations in sarcoidosis:a case/control association study. Eur. J. Hum. Genet. 8, 717-720 (2000).

4 Ravnik-Glavac, M., Dean, M., di Sant'Agnese, P., Chernick, M., Kozelj, M., Krizman, I. et al. Evidence that hereditary pancreatitis is genetically heterogeneous disorder. Pflugers Arch. 439, R50-R52 (2000).

5 Tzetis, M., Efthymiadou, A., Strofalis, S., Psychou, P., Dimakou, A., Pouliou, E. et al. CFTR gene mutations-including three novel nucleotide substitutions-and haplotype background in patients with asthma, disseminated bronchiectasis and chronic obstructive pulmonary disease. Hum. Genet. 108, 216-221 (2001).

6 Divac, A., Nikolic, A., Mitic-Milikic, M., Nagorni-Obradovic, L., Petrovic-Stanojevic, N., Dopudja-Pantic, V. et al. High frequency of theR75Q CFTR variation in patients with chronic obstructive pulmonary disease. J. Cyst. Fibros. 3, 189-191 (2004).

7 Cohn, J. A., Neoptolemos, J. P., Feng, J., Yan, J., Jiang, Z., Greenhalf, W. et al. Increased risk of idiopathic chronic pancreatitis incystic fibrosis carriers. Hum. Mutat. 26, 303-307 (2005)

8 Schneider, A., Larusch, J., Sun, X., Aloe, A., Lamb, J., Hawes, R. et al. Combined bicarbonate conductance-impairing variants in CFTR and SPINK1 variants are associated with chronic pancreatitis in patients without cystic fibrosis. Gastroentero/ogy 140, 162-171 (2011).

9 Aissat, A., De Becdelievre, A., Golmard, L., Vasseur, C., Costa, C., Chaoui, A. et al. Combined computational-experimental analyses of CFTR exon strength uncover predictability of exon-skipping level. Hum. Mutat. 34, 873-881 (2013).

10 Rosendahl, J., Landt, O., Bernadova, J., Kovacs, P., Teich, N., Bödeker, H. et al. CFTR, SPINK1, CTRC and PRSS1 variants in chronic pancreatitis: is the role of mutated CFTR overestimated? Gut 62, 582-592 (2013).

11 Bobadilla, J. L., Macek, M. Jr, Fine, J. P. \& Farrell, P. M. Cystic fibrosis: a worldwide analysis of CFTR mutations - correlation with incidence data and application to screening. Hum. Mutat. 19, 575-606 (2002). 
12 Nikolic, A., Divac, A., Stankovic, M., Dinic, J., Tomic, B. \& Ljujic, M. Analysis ofcommon CFTR polymorphisms 5T, M470V and R75Q in healthy Serbian population. Genetika 42, 996-998 (2006).

13 Bishop, M. D., Freedman, S. D., Zielenski, J., Ahmed, N., Dupuis, A., Martin, S. et al. The cystic fibrosis transmembrane conductance regulator gene and ion channel function in patients with idiopathic pancreatitis. Hum. Genet. 118 , 372-381 (2005)

14 Cohn, J. A., Neoptolemos, J. P., Feng, J., Yan, J., Jiang, Z., Greenhalf, W. et al. Increased risk of idiopathic chronic pancreatitis in cystic fibrosis carriers. Hum. Mutat. 26, 303-307 (2005).

15 Weiss, F. U., Simon, P., Bogdanova, N., Mayerle, J., Dworniczak, B., Horst, J. et al. Complete cystic fibrosis transmembrane conductance regulator gene sequencing in patients with idiopathic chronic pancreatitis and controls. Gut 54, 1456-1460 (2005).

16 Ockenga, J., Stuhrmann, M., Ballmann, M., Teich, N., Keim, V., Dörk, T. et al. Mutations of the cystic fibrosis gene, but not cationic trypsinogen gene, are associated with recurrent or chronic idiopathic pancreatitis. Am. J. Gastroenterol. 95, 2061-2067 (2000)

17 Dörk, T., Dworniczak, B., Aulehla-Scholz, C., Wieczorek, D., Böhm, I., Mayerova, A. et al. Distinct spectrum of CFTR gene mutations in congenital absence of vas deferens. Hum. Genet. 100, 365-377 (1997).

18 Masson, E., Chen, J. M., Audrezet, M. P., Cooper, D. N. \& Férec, C. A conservative assessment of the major genetic causes of idiopathic chronic pancreatitis: Data from a comprehensive analysis of PRSS1, SPINK1, CTRC and CFTR genes in 253 young French patients. PLoS One 8, e73522 (2013).

19 Tzetis, M., Kaliakatsos, M., Fotoulaki, M., Papatheodorou, A., Doudounakis, S., Tsezou, A. et al. Contribution of the CFTR gene, the pancreatic secretory trypsin inhibitor gene (SPINK1) and the cationic trypsinogen gene (PRSS1) to the etiology of recurrent pancreatitis. Clin. Genet. 71, 451-457 (2007).

20 Bombieri, C., Benetazzo, M., Saccomani, A., Belpinati, F., Gilè, L. S., Luisetti, M. et al. Complete mutational screening of the CFTR gene in 120 patients with pulmonary disease. Hum. Genet. 103, 718-722 (1998).

21 Divac, A., Nikolic, A., Mitic-Milikic, M., Nagorni-Obradovic, L., Petrovic-Stanojevic, N., Dopudja-Pantic, V. et al. High frequency of the R75Q CFTR variation in patients with chronic obstructive pulmonary disease. J. Cyst. Fibros. 3, 189-191 (2004).

22 Ziedalski, T. M., Kao, P. N., Henig, N. R., Jacobs, S. S. \& Ruoss, S. J. Prospective analysis of cystic fibrosis transmembrane regulator mutations in adults with bronchiectasis or pulmonary nontuberculous mycobacterial infection. Chest 130, 9951002 (2006).

23 Divac, A., Nikolic, A., Mitic-Milikic, M., Nagorni-Obradovic, L., Petrovic-Stanojevic, N. Dopudja-Pantic, V. et al. CFTR mutations and polymorphisms in adults with disseminated bronchiectasis: a controversial issue. Thorax 60, 85 (2005).

24 Girodon, E., Cazeneuve, C., Lebargy, F., Chinet, T., Costes, B., Ghanem, N. et al. CFTR gene mutations in adults with disseminated bronchiectasis. Eur. J. Hum. Genet. 5, 149-155 (1997)

25 Bombieri, C., Luisetti, M., Belpinati, F., Zuliani, E., Beretta, A., Baccheschi, J. et al. Increased frequency of CFTR gene mutations in sarcoidosis: a case/control association study. Eur. J. Hum. Genet. 8, 717-720 (2000).

26 Noone, P. G., Zhou, Z., Silverman, L. M., Jowell, P. S., Knowles, M. R. \& Cohn, J. A Cystic fibrosis gene mutations and pancreatitis risk: relation to epithelial ion transport and trypsin inhibitor gene mutations. Gastroenterology 121, 1310-1319 (2001).

27 Li, W., Soave, D., Miller, M. R., Keenan, K., Lin, F., Gong, J. et al. Unraveling the complex genetic model for cystic fibrosis: pleiotropic effects of modifier genes on early cystic fibrosis-related morbidities. Hum. Genet. 133, 151-161 (2014). 